\title{
Motion-Sensitive Responses in Visual Area V4 in the Absence of Primary Visual Cortex
}

\author{
Michael C. Schmid, ${ }^{1 \star}$ Joscha T. Schmiedt, ${ }^{1 \star}$ Andrew J. Peters, ${ }^{2}$ Richard C. Saunders, ${ }^{4}$ Alexander Maier, ${ }^{3}$ \\ and David A. Leopold ${ }^{4,5}$ \\ ${ }^{1}$ Ernst Strüngmann Institute for Neuroscience in cooperation with Max Planck Society, 60528 Frankfurt, Germany, ${ }^{2}$ University of California San Diego, La \\ Jolla, California 92093-0634, ${ }^{3}$ Vanderbilt University, Department of Psychology, Nashville, Tennessee 37240, ${ }^{4}$ Laboratory of Neuropsychology, National \\ Institute of Mental Health, Bethesda, Maryland 20892, and ${ }^{5}$ Neurophysiology Imaging Facility, National Institute of Mental Health, National Institute of \\ Neurological Disorders and Stroke, and National Eye Institute, Bethesda, Maryland 20892
}

Neurons in cortical ventral-stream area V4 are thought to contribute to important aspects of visual processing by integrating information from primary visual cortex (V1). However, how V4 neurons respond to visual stimulation after V1 injury remains unclear: While electrophysiological investigation of V4 neurons during reversible V1 inactivation suggests that virtually all responses are eliminated (Girard et al., 1991), fMRI in humans and monkeys with permanent lesions shows reliable V1-independent activity (Baseler et al., 1999; Goebel et al., 2001; Schmid et al., 2010). To resolve this apparent discrepancy, we longitudinally assessed neuronal functions of macaque area V4 using chronically implanted electrode arrays before and after creating a permanent aspiration lesion in V1. During the month after lesioning, we observed weak yet significant spiking activity in response to stimuli presented to the lesion-affected part of the visual field. These V1-independent responses showed sensitivity for motion and likely reflect the effect of V1-bypassing geniculate input into extrastriate areas.

\section{Introduction}

The visual system is thought to operate via serially and hierarchically organized processes (Felleman and van Essen, 1991) where damage to earlier stages leads to loss of function in later stages. However, at the level of primary visual cortex (V1), lesions do not abolish all visual function: Humans and nonhuman primates can retain residual vision in the form of "blindsight" (Cowey, 2010), a term that was coined to describe the ability to detect and respond to visual stimuli in the absence of conscious visual experience. Research over the past years has established that activity in higher cortical areas, most prominently in motion-sensitive area V5/MT, is correlated with blindsight (Rodman et al., 1989; Zeki and Ffytche, 1998; Baseler et al., 1999; Goebel et al., 2001; Azzopardi et al., 2003; Schmid et al., 2010). However, the extent to which ventral stream cortex retains responsiveness without V1 input remains controversial. Here we address this question at the

Received Sept. 12, 2013; revised 0ct. 15, 2013; accepted 0ct. 19, 2013.

Author contributions: M.C.S., A.M., and D.A.L. designed research; M.C.S., A.J.P., R.C.S., A.M., and D.A.L. performed research; M.C.S. and J.T.S. analyzed data; M.C.S., J.T.S., and D.A.L. wrote the paper.

This work was supported by the Intramural Research Program of the National Institute of Mental Health (D.A.L.) and a Deutsche Forschungsgemeinschaft Emmy Noether grant (M.C.S.). The authors thank Alex Cummings, David Hu, Charles Zhu, Frank Ye, George Dold, Andy Mitz, Rachel Reoli, Katy Smith, and James Yu for technical assistance; Michele Cox for help with experiments and analysis; Georgios Spyropoulos for preliminary analysis; and Pascal Fries for support.

The authors declare no competing financial interests.

${ }^{*}$ M.C.S. and J.T.S. contributed equally to this work.

Correspondence should be addressed to Dr. Michael C. Schmid, Ernst Strüngmann Institute for Neuroscience in cooperation with Max Planck Society, Deutschordenstrasse 46, 60528 Frankfurt, Germany. E-mail: michael.schmid@esi-frankfurt.de.

DOI:10.1523/JNEUROSCI.3923-13.2013

Copyright $(2) 2013$ the authors $\quad 0270-6474 / 13 / 3318740-06 \$ 15.00 / 0$ level of V4, an area thought to be primarily important for shape and object processing (Roe et al., 2012). Previous assessment of neuronal activity during temporary V1 cooling under anesthetized conditions concluded that V4 is entirely dependent on V1 input (Girard et al., 1991). However, this finding is in apparent conflict with reports of reliable fMRI responses in V4 of awake monkeys and humans with chronic V1 lesions (Baseler et al., 1999; Goebel et al., 2001; Schmid et al., 2010). These V1independent fMRI responses were shown to depend on the lateral geniculate nucleus (LGN) of the thalamus (Schmid et al., 2010) and thus most likely rely on a geniculo-extrastriate pathway (Rodman et al., 2001; Sincich et al., 2004) that draws its input from the superior colliculus (Harting et al., 1991). To resolve the apparent conflict between electrophysiology and fMRI and to control for some of the methodological differences that may have contributed to these discrepant findings, we chose an approach in which we longitudinally recorded the multiunit spiking activity (MUA) in behaving monkeys before and after permanently lesioning V1.

\section{Materials and Methods}

Subjects. Two healthy adult female rhesus monkeys (Macaca mulatta, Monkey B and Monkey F) with prior V1 lesions in the right hemisphere were used in the study. All procedures were in accordance with the Institute for Laboratory Animal Research Guide for the Care and Use of Laboratory Animals and approved by the Animal Care and Use Committee of the National Institute of Mental Health.

Surgical procedures. A large occipital bone flap over the left hemisphere was created to warrant access to areas V1 and V4. A chronic $10 \times 10$ "Utah" array of microelectrodes (Blackrock Microsystems) was subdurally inserted on the prelunate gyrus, $\sim 2 \mathrm{~mm}$ dorsal of the lateral tip of the 
A
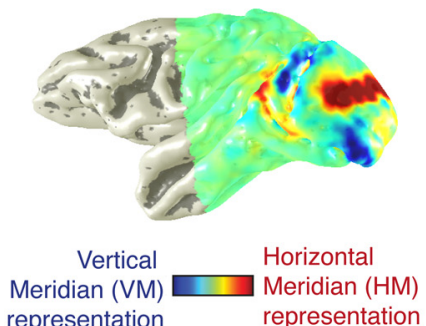

B

D

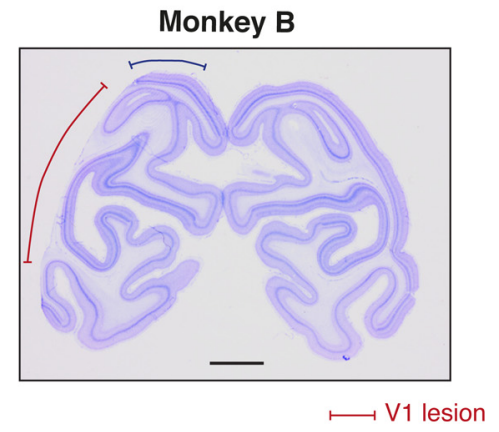

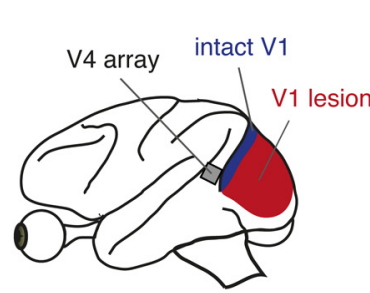

V4 array implantation V1 lesion
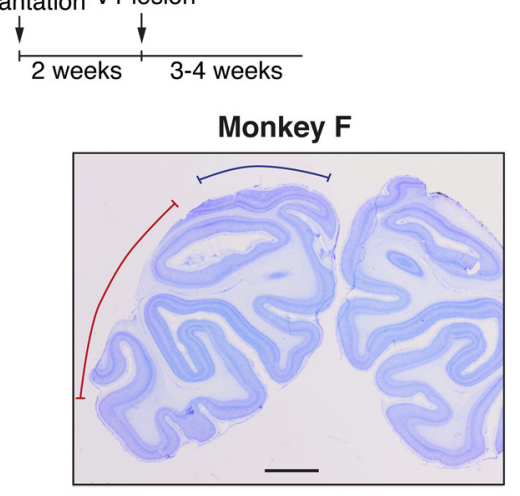

$\longrightarrow$ intact $\mathrm{V} 1$

Figure 1. Longitudinal investigation of V4 neuronal responses before and after V1 lesion. $\boldsymbol{A}$, fMRI-based retinotopic map of visual cortex for Monkey B, acquired before lesioning V1 using alternating rotating checkerboard wedges. $\boldsymbol{B}$, The lesion was targeted to eliminate the V1 representation of the right horizontal meridian between $\sim 2-7^{\circ}$ of visual eccentricities (red) while leaving lower vertical meridian representation intact (blue). C, Stimuli close to the right horizontal meridian inside the lesionaffected visual space are labeled "scotoma stimuli" and stimuli close to the vertical meridian are labeled "control stimuli." $\boldsymbol{D}$, Coronal section of V1 for Monkey B (left) and F (right). Scale bars, $5 \mathrm{~mm}$.

inferior occipital sulcus. Each microelectrode was $1.5 \mathrm{~mm}$ long with a tip radius ranging from 3 to $5 \mu \mathrm{m}$, and an interelectrode spacing of $400 \mu \mathrm{m}$. After array implantation, dura and bone flap were sutured back in place and covered with the skin. In a subsequent surgery, following the first period of recordings, V1 was lesioned using procedures as described previously (Schmid et al., 2010).

Behavioral task and visual stimulation. Eye movements were monitored either using the scleral search coil technique or via infrared-based tracking of the pupil. To delineate the outline of the visual deficit ("scotoma") in the part of the visual field containing the V4-receptive fields, monkeys were trained on a simple perimetry task before lesioning. To this end, monkeys learned to initially fixate a small centrally placed dot $\left(0.4^{\circ}\right.$ diameter $)$. If the monkeys maintained fixation for $500 \mathrm{~ms}$ within maximally $1.5-2^{\circ}$ diameter of the fixation spot, a second dot $\left(0.4^{\circ} \mathrm{diam}-\right.$ eter) was presented in addition to the fixation spot for $1000-2000 \mathrm{~ms}$ in one of $10 \times 10$ possible target positions of a virtual grid in the lower right visual field quadrant $\left(-1^{\circ}\right.$ to $9^{\circ}$, grid resolution $\left.1^{\circ}\right)$. To receive reward, monkeys had to execute a single saccade and acquire fixation for $500 \mathrm{~ms}$ within a new fixation window of $2^{\circ}$ diameter around the target.

To probe for visually elicited neuronal responses, monkeys were trained to maintain passive fixation within $1.5-2^{\circ}$ diameter of a fixation spot while the various visual stimuli were displayed on the screen. Visual stimuli were generated using OpenGL-based custom written software (ESS/STIM, courtesy of Dr. D. Sheinberg, Brown University) running on industrial PCs (Kontron) with NVIDIA Quadro FX 3000 graphics boards. Stimuli were presented with a screen refresh rate of $60 \mathrm{~Hz}$ on a single LCD Samsung monitor (height $40 \mathrm{~cm}$, width $65 \mathrm{~cm}$ ) positioned at a viewing distance of $100 \mathrm{~cm}$. All stimuli were presented for $500 \mathrm{~ms}$ on a dark gray background. Typical stimulus presentations per session were between 1000 and 1300 .

For receptive field mapping, random dot kinematographs with a diameter of $1-1.5^{\circ}$ were presented at pseudo-randomly selected stimulus locations on an $8 \times 8$ virtual grid in the lower right visual field quadrant $\left(0^{\circ}-7^{\circ}\right.$, grid resolution $\left.1^{\circ}\right)$. The direction of dot movement (upward or downward) was randomly varied on a trial-by-trial basis while the moCStimulus
Position

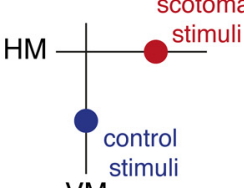

VM tion strength was kept constant at $100 \%$ dot coherence. Individual dots were white and moved with a speed of $6 \%$.

Grating stimuli had a diameter of $1^{\circ}$ or $1.5^{\circ}$ and were displayed at either one of the following positions: $\left(0^{\circ},-3.5^{\circ}\right),\left(3^{\circ},-3^{\circ}\right),\left(3.5^{\circ}, 0^{\circ}\right)$. The first and the last of these positions were used as control and scotoma stimulus in this study, respectively. The gratings were either static or slowly drifting at 0.3 or $0.5 \%$ s and varied with respect to spatial frequency $\left(0.7-10^{\circ} \%\right.$ s), orientation $\left(0^{\circ}, 90^{\circ}, 180^{\circ}, 270^{\circ}\right.$ with two of these orientations resulting in equal stimuli for static gratings), and luminance contrast (low vs high, only high contrast considered here).

For the assessment of directional motion tuning, random dot kinematograms with four randomly selected directions of motion $\left(0^{\circ}\right.$, $\left.90^{\circ}, 180^{\circ}, 270^{\circ}\right)$ were presented at the same stimulus positions as the gratings described above. They varied with respect to coherence (10\%, $50 \%$, or $100 \%$, only $100 \%$ considered here) and color (red, green, blue, or yellow, all considered here). Speed and size were the same as for the receptive field mapping.

Neurophysiological recordings. Electrical reference was a wire located subdurally over the parietal cortex. We recorded from a random selection of 64 channels of the 96 channels available from the array. Electrode impedances ranged between 150 and $1 \mathrm{M} \Omega$ at $1 \mathrm{kHz}$. Extracellular voltages were amplified, filtered between $0.1 \mathrm{~Hz}$ and $12 \mathrm{kHz}$, and digitized at 24,414.1 Hz using a 64-channel RZ2 recording system (Tucker Davis Technologies).

Data analysis. All data were analyzed with the MATLAB (R2011a, MathWorks) toolbox FieldTrip (Oostenveld et al., 2011) and customwritten scripts. To obtain an estimate of MUA, the field potential was bandpass-filtered between $300 \mathrm{~Hz}$ and $12 \mathrm{kHz}$, rectified, low-pass filtered at $120 \mathrm{~Hz}$ with a fourth order zero-phase Butterworth filter, and downsampled to $256 \mathrm{~Hz}$, yielding a quasi-continuous measure of high-frequency field power. Data from each trial were then normalized to their respective $250 \mathrm{~ms}$ prestimulus period and expressed as percentage change from baseline throughout the paper. Data were pooled across sessions, except for the direction of motion analysis. Responses were estimated by averaging the normalized MUA signal between 50 and $500 \mathrm{~ms}$. For presentation purposes, the MUA time courses were smoothed by convolution with a Gaussian kernel of 125 $\mathrm{ms} \mathrm{SD}$. The array receptive field (RF) was determined by averaging the individual receptive fields of all visually responsive electrodes and interpolating to a resolution of $0.5^{\circ}$. Tuning for direction of motion was assessed by comparing the average responses of the preferred feature (i.e., the one resulting in strongest response) to the nonpreferred feature (i.e., with minimal response). Tuning strength is quantified with a tuning index $d^{\prime}$ as follows:

$$
d^{\prime}=\frac{\mathrm{MUA}_{\mathrm{PF}}-\mathrm{MUA}_{\mathrm{nonPF}}}{\hat{\sigma}}
$$

where $\hat{\sigma}=\sqrt{\left(\sigma_{\mathrm{PF}}^{2}+\sigma_{\mathrm{nonPF}}^{2}\right) / 2}$ is the pooled SD of the two response distributions. Significance was assessed by comparing actual $d^{\prime}$ values against a surrogate distribution obtained by randomly shuffling trial labels 10,000 times.

\section{Results}

To delineate the effect of permanent V1 lesions on electrophysiological responses in macaque ventral-stream visual cortex, we longitudinally monitored V4 MUA during the presentation of various spatially restricted stimuli over a period of several weeks 
A

\section{Before V1 lesion}

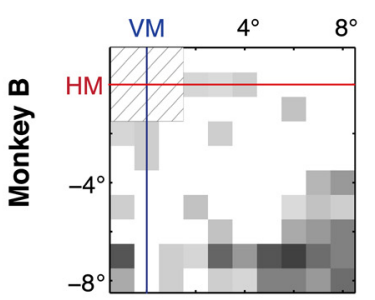

B
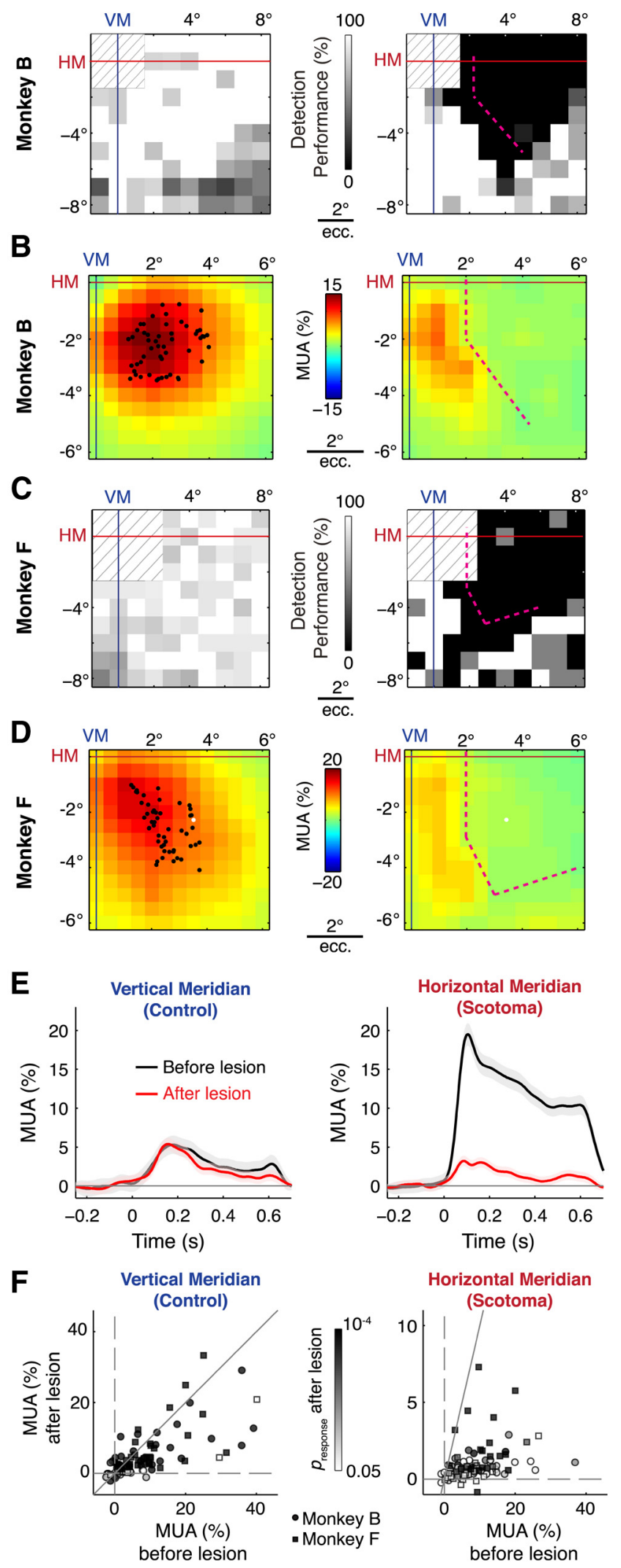

Figure 2. V4 responses to scotoma stimuli are severely degraded but not abolished. $\boldsymbol{A}$, Detection performance of Monkey $B$ in a perimetry task at various stimulus positions in the lower right visual field during the week before (left, $n=532$ trials from 2 sessions) and after (right, $n=705$ trials from 3 sessions) the V1 lesion. Dashed magenta line indicates scotoma using chronically implanted multielectrode arrays (Fig. 1). Following detailed presurgical retinotopic mapping by fMRI (Fig. $1 A$ ), we first implanted electrode arrays into area V4 at a position where the average receptive field of neurons was centered at $\sim 3^{\circ}$ of eccentricity. After $\sim 2$ weeks of initial electrophysiological assessment with V1 intact, we carried out permanent V1 lesions by aspirating V1's gray matter from the representation of the fovea laterally up to $\sim 7^{\circ}$ medially, straddling most of the upper and lower visual field representations around the horizontal meridian (Fig. 1B). Importantly, however, a section of approximately 5 $\mathrm{mm}$ near the lunate sulcus representing the visual vertical meridian (including the boundary to area V2) was not aspirated to allow for control conditions with V1 intact (Fig. $1 B, C$ ). For the experiments described below, this enabled us to compare neuronal responses with V1 intact versus V1 lesioned, regardless of the time point of assessment (Fig. 1C). Postmortem histological assessment of the brains of both monkeys confirmed complete loss of gray matter in the lesioned area of V1 and the preservation of the region of cortex that served as a control in the experiments (Fig. 1D).

\section{Behavioral performance}

During the week after the lesion, we assessed the animals' behavioral ability to detect visual stimuli and recorded V4 spiking responses to visual stimuli presented at different positions in the visual field. As expected, the V1 lesion resulted in a circumscribed scotoma: there was a severe deficit in detecting visual stimuli in a zone surrounding the horizontal meridian, whereas vision at the vertical meridian remained largely intact (Fig. $2 A, C$ ).

Spatial organization of V4 responses with respect to scotoma We next assessed the effects of the V1 lesion on the large-scale visuotopic RF organization of the sampled V4 population by presenting stimuli at various locations in the lower right visual field. The longitudinal design of our study allowed for a direct comparison of the RFs from precisely the same cortical location before and after the lesion. In both monkeys, the array RF (i.e., the average RF across all visually responsive electrodes) showed a strong reduction in response amplitude for stimuli around the horizontal meridian that closely matched the behavioral scotoma (compare Fig. $2 A, C$ with Fig. $2 B, D$, respectively), whereas the responses close to the vertical meridian remained mostly intact. On average, MUA responses to stimuli presented near the horizontal meridian were reduced from $8 \pm 1.0 \%$ before the lesion to $0.70 \pm 0.076 \%$ after the lesioning Monkey B $\left(n=53\right.$ electrodes, $p<10^{-9}$, Wilcoxon signed rank

border estimate. $\boldsymbol{B}$, V4 MUA array RF of Monkey B before (left, $n=3524$ trials from $n=5$ sessions) and after (right, $n=4882$ trials from $n=7$ sessions) V1 lesion ( $n=53$ electrodes). Black dots indicate RF centers of individual electrodes. $\boldsymbol{C}$, Detection performance as in $\boldsymbol{A}$ for Monkey F before ( $n=1344$ trials from 3 sessions) and after V1 lesion ( $n=206$ trials from 1 session). $\boldsymbol{D}, \mathrm{V} 4$ array RF as in $\boldsymbol{B}$ for Monkey $\mathrm{F}(n=47$ electrodes, $n=2193$ and 4530 trials from $n=3$ and 6 sessions from before and after V1 lesion, respectively). White dot indicates RF center of example electrode shown in $\boldsymbol{E}$. $\boldsymbol{E}$, MUA response from example electrode with large RF coverage in Monkey $\mathrm{F}$ to stimuli close to vertical meridian (left, $x=0$ to $1^{\circ}, y=-3$ to $-4^{\circ}$ ) and horizontal meridian (right, $x=3$ to $4^{\circ}, y=-2$ to $0^{\circ}$ ) before and after V1 lesion. Shading indicates SEM. $F$, Distribution of MUA responses to stimuli close to vertical meridian (left) and horizontal meridian (right). Each dot indicates the average MUA response of a recording site before and after V1 lesion. Color shading indicates $p$ value of postlesion responses (Wilcoxon signed rank test compared with prestimulus period). Solid gray lines indicate identical prelesion and postlesion responses. ecc., Eccentricity. 
A
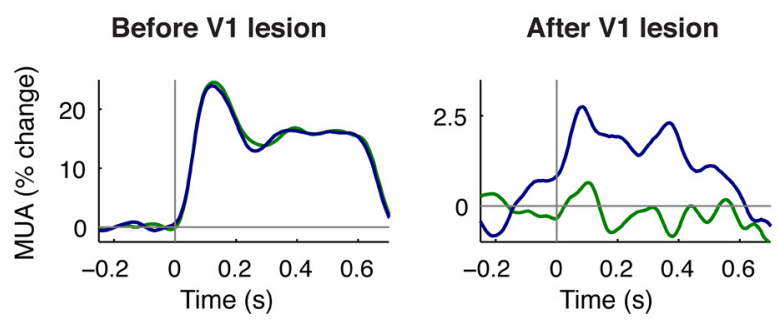

B
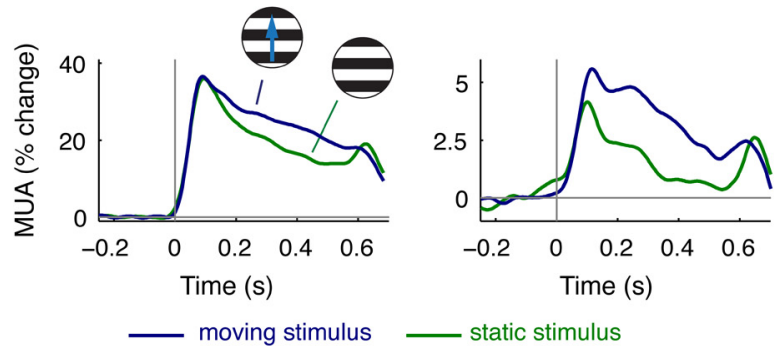

C

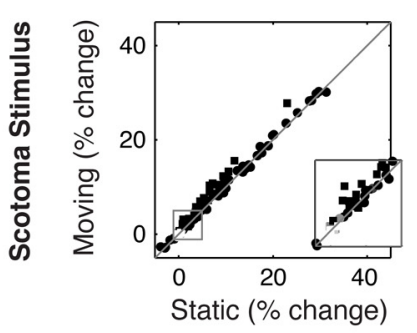

After V1 lesion
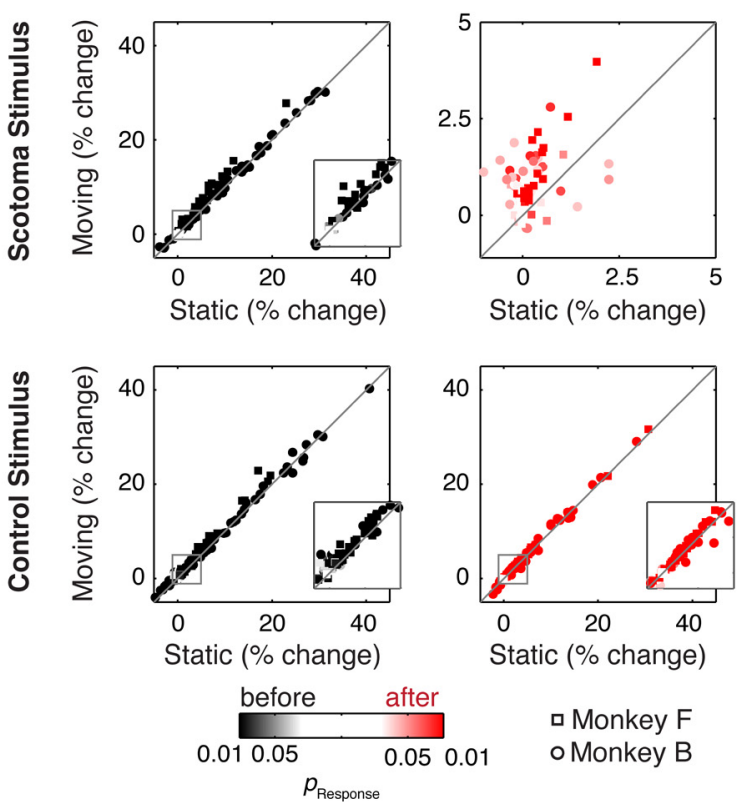

口 Monkey $\mathrm{F}$

o Monkey B

Figure 3. V4 MUA responses to scotoma stimuli show sensitivity for motion. $\boldsymbol{A}, \boldsymbol{B}$, Example MUA time courses for moving (blue) and static (green) gratings before (left) and after (right) V1 lesion from Monkey $B(\boldsymbol{A})$ and Monkey $F(\boldsymbol{B})$. C, Distribution of responses to moving and static gratings of all recording sites for scotoma (top row) and control (bottom row) stimuli. Each dot represents the average MUA response of a recording site, before the lesion (black symbols, left column; Monkey B: $n=7$ sessions; Monkey F: $n=4$ sessions) or after (red symbols, right column; Monkey B: $n=3$ sessions, $5-9 \mathrm{~d}$ after lesion; Monkey $\mathrm{F}: n=5$ sessions, $9-18 \mathrm{~d}$ after lesion). Insets, Magnified view of the gray box.

test) and from $8.4 \pm 0.87 \%$ before lesioning to $1.1 \pm 0.23 \%$ after lesioning in Monkey $\mathrm{F}\left(n=47\right.$ electrodes, $p<10^{-8}$ ). Despite these greatly reduced activity levels in V1 after the V1 lesion, several sites continued to show significant MUA responses to visual stimuli presented to the scotoma (e.g., see Fig. 2E): 26 of 43 and 33 of 46 electrodes in Monkey B and F, respectively, remained responsive to visual stimulation close to the horizontal meridian $(p<0.05$, Wilcoxon signed rank test compared with baseline). In contrast, for stimuli close to the vertical meridian (the control region outside the scotoma), virtually all recording sites remained responsive ( 39 of 39 electrodes in Monkey B, 42 of 47 in Monkey F).
A
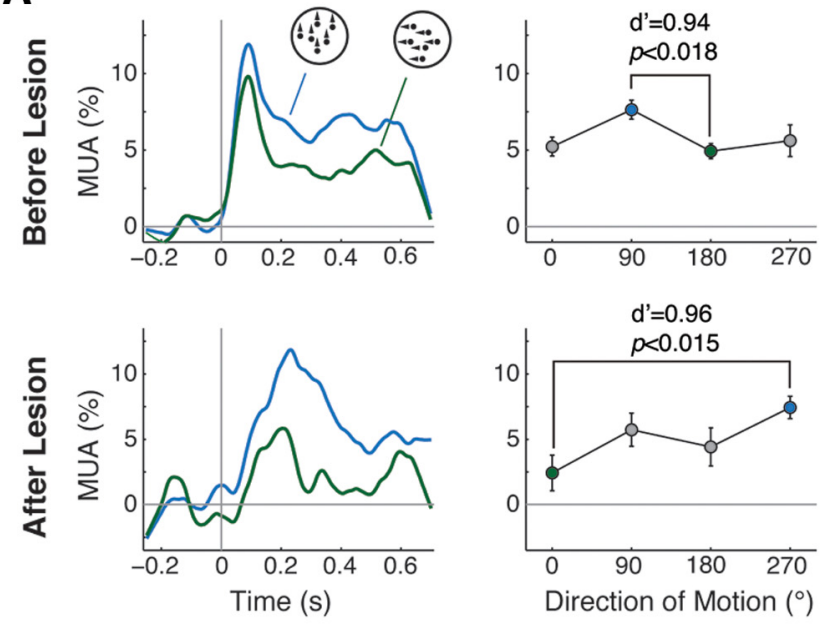

B

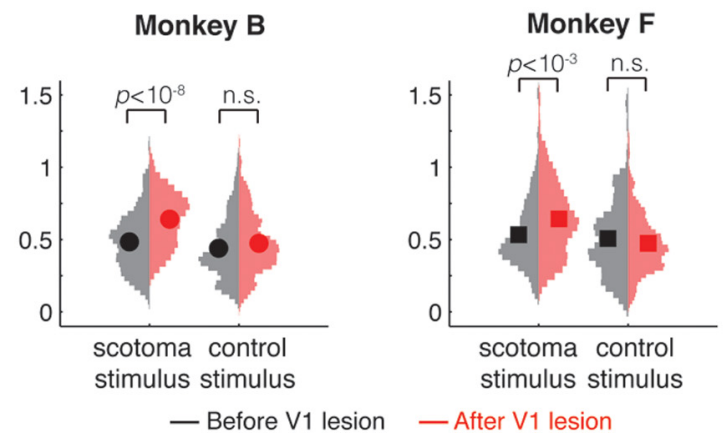

Figure 4. V1-independent tuning for direction of motion in V4. A, MUA time courses (left column) and tuning profile (right column) from example sessions before (top row) and after (bottom row) V1 lesion of Monkey F. Tuning is quantified by taking the $d^{\prime}$ value between the maximum (blue) and minimum (green) MUA response. Error bars indicate SEM. B, Smoothed histograms of $d^{\prime}$ values for direction of motion tuning from all responsive recording sites and sessions before (gray, Monkey B: $n=5$ sessions, Monkey F: $n=3$ sessions) and after (red, Monkey B: $n=4$ sessions, Monkey F: $n=11$ sessions) the V1 lesion for scotoma or control stimuli. Larger symbols indicate mean values. n.s. indicates a difference not significant at $p<$ 0.05 (Mann-Whitney U test).

\section{Motion sensitivity of residual neuronal responses to scotoma} restricted stimuli

The residual responses to stimuli in the lesion-affected visual space demonstrate V1-independent transmission of visual information to area V4. To further characterize the nature of these responses with respect to the preserved motion detection that characterizes the phenomenon of blindsight after V1 injury (Zeki and Ffytche, 1998), we assessed the extent of neural motion sensitivity in our V4 sample. In a first step, we compared V4 responses to moving versus static gratings (Fig. 3). After the lesion, almost all of the recording sites with residual neuronal responses (MUA $>0.05 \%$ and $p<0.05$ Wilcoxon signed rank test against prestimulus period) of both monkeys revealed a preference for moving gratings (Fig. 3C, top right; Monkey B: $p<0.04, n=11$ sites; Monkey F: $p<0.0012, n=18$ sites, Wilcoxon signed rank test), indicating a residual sensitivity of V1-deafferented V4 neurons for visual motion.

To test whether V4's preference for moving stimuli after lesioning V1 includes directional tuning, we analyzed spiking responses to random dot patterns that coherently moved in one of four different directions of motion (Fig. 4). Before the V1 lesion, 16 of $190(8.4 \%)$ and 11 of $106(10.4 \%)$ recordings from responsive sites (MUA $>1 \%$ and $p<0.05$, Wilcoxon signed rank test) 
in Monkey B and Monkey F, respectively, showed significant $(p<0.05$, trial label shuffling test, see Materials and Methods) tuning for direction of motion (Fig. $4 A$, top). Surprisingly, significant directional tuning could also be observed after V1 removal (Fig. $4 A$, bottom). Indeed, the number of significantly motion-tuned V4 sites increased, resulting in 19 of 81 (23.5\%) and 30 of 129 (23.3\%) significantly tuned recordings in Monkey $\mathrm{B}$ and Monkey F, respectively. To further quantify the tuning strength, we calculated $d^{\prime}$ between the multiunit responses of the preferred and the least preferred stimulus (Fig. $4 A$ ). In both monkeys, $d^{\prime}$ for motion direction increased after the V1 lesion (Fig. $4 B$, Mann-Whitney U test), yet only for the scotoma-directed stimuli and not for the control stimuli (Fig. 4B), thus highlighting the dependence on absent V1 input for this effect.

\section{Discussion}

Our results demonstrate visually driven V4 neuronal responses in the absence of V1 input, which are sensitive for stimulus motion. We discuss these findings in the context of V4's role in motion processing, the pathways that may contribute to V1-independent visual functions, and the implications for blindsight.

\section{Motion processing in V4: dependence on V1 input and plasticity}

V4 neurons have been implicated in a wide range of stimulus features, including orientation, color, shape, and motion (Roe et al., 2012). In our experiments, we have assessed motion sensitivity in V4 with and without V1 input. With respect to the strength of motion selectivity, our measures with V1 intact are at the lower end of the reported effects for V4 (Desimone and Schein, 1987; Ferrera et al., 1994; Tolias et al., 2005). However, as recent data from optical imaging suggest a modular organization of V4 with discreet patches of cortex dedicated to motion tuning (Li et al., 2013), it is conceivable that our electrode arrays may have undersampled these patches. Such a potential bias notwithstanding, we used a longitudinal recording approach, which permitted us to sample from the same cortical tissue at multiple time points before and after lesioning V1. We found increased directional selectivity in both the number of tuned electrode sites as well as in the $d^{\prime}$ values (Fig. $4 B$ ) when there was no input from V1 to V4. It is possible that this increased sensitivity is the result of local unmasking: the balance of neurons that are interconnected via inhibitory synapses but are tuned to different stimulus features due to diverse inputs might have shifted toward motion processing after the V1 lesion. As this plastic process may take some time to take effect, it could explain the differences between previous electrophysiological assessments of V4 activity during V1 cooling (Girard et al., 1991) and the ones by fMRI under chronic lesion conditions (Baseler et al., 1999; Goebel et al., 2001; Schmid et al., 2010).

\section{V1-independent pathways to V4}

The observation of V1-independent responses to visual stimuli in area V4 implies a subcortical pathway that bypasses V1 and relays information from the retina to visual association cortex. The superior colliculus, which receives direct retinal input and whose neurons exhibit motion sensitivity (Marrocco and Li, 1977), is a likely contributor to such a pathway projecting directly to the visual thalamus (Harting et al., 1991). This projection is often considered to be a component of one or more secondary visual pathways that circumvent V1 to reach extrastriate cortex, including areas $\mathrm{V} 4$ and $\mathrm{V} 5 / \mathrm{MT}$, via relays in LGN and pulvinar (Benevento and Rezak, 1976; Benevento and Yoshida, 1981; Ly- sakowski et al., 1988; Rodman et al., 2001). Although the role of the pulvinar for V1-independent processing remains uncertain, the superior colliculus and LGN have been demonstrated to be critical structures for mediating blindsight-related effects (Mohler and Wurtz, 1977; Rodman et al., 1990; Schmid et al., 2010; Kato et al., 2011). Alternatively, or more likely in addition to the direct subcortical route to $\mathrm{V} 4$, intracortical projections from motion-sensitive area V5/MT to V4 may contribute to the effects we observed (Maunsell and van Essen, 1983). It is attractive to implicate V5/MT in mediating the responses we observed in V4 due to the robust input V5/MT receives from the LGN (Sincich et al., 2004), as well as MT's prominent motion sensitivity (Albright et al., 1984). Input from higher areas other than V5/MT remains also a viable option for contributing to our observations in V4.

\section{Implications for blindsight}

The term "blindsight" was coined by Weiskrantz and colleagues to describe visual capacities that survive V1 injury that exist in the absence of conscious visual experience (Cowey, 2010). A prominent feature of blindsight in both humans and monkeys is the ability to detect moving stimuli (Zeki and Ffytche, 1998). Typically, the preserved sensitivity for motion in blindsight has been associated with area V5/MT because of this area's high proportion of motion-selective neurons (Albright et al., 1984) and because of several reports of preserved brain activity in MT after V1 damage (Rodman et al., 1989; Zeki and Ffytche, 1998; Azzopardi et al., 2003). The remarkable motion capacities that survive V1 injury are, however, in strong contrast with the deteriorating deficits of a V1 lesion with respect to color or shape judgments (Cowey, 2010), functions that are typically associated with the cortical ventral stream, including area V4 (Roe et al., 2012). Whereas for the present data the monkeys' V1-independent detection capacities remain unclear, our electrophysiological results appear to be in good agreement with the general phenotype of blindsight.

Together, our findings lend neurophysiological support for previous observations made by fMRI of extrastriate visual responses after V1 damage (Baseler et al., 1999; Goebel et al., 2001; Schmid et al., 2010): V4 spiking can be visually driven in the absence of V1 input.

\section{References}

Albright TD, Desimone R, Gross CG (1984) Columnar organization of directionally selective cells in visual area MT of the macaque. J Neurophysiol 51:16-31. Medline

Azzopardi P, Fallah M, Gross CG, Rodman HR (2003) Response latencies of neurons in visual areas MT and MST of monkeys with striate cortex lesions. Neuropsychologia 41:1738-1756. CrossRef Medline

Baseler HA, Morland AB, Wandell BA (1999) Topographic organization of human visual areas in the absence of input from primary cortex. J Neurosci 19:2619-2627. Medline

Benevento LA, Rezak M (1976) The cortical projections of the inferior pulvinar and adjacent lateral pulvinar in the rhesus monkey (Macaca mulatta): an autoradiographic study. Brain Res 108:1-24. CrossRef Medline

Benevento LA, Yoshida K (1981) The afferent and efferent organization of the lateral geniculo-prestriate pathways in the macaque monkey. J Comp Neurol 203:455-474. CrossRef Medline

Cowey A (2010) The blindsight saga. Exp Brain Res 200:3-24. CrossRef Medline

Desimone R, Schein SJ (1987) Visual properties of neurons in area V4 of the macaque: sensitivity to stimulus form. J Neurophysiol 57:835-868. Medline

Felleman DJ, van Essen DC (1991) Distributed hierarchical processing in the primate cerebral cortex. Cereb Cortex 1:1-47. CrossRef Medline

Ferrera VP, Rudolph KK, Maunsell JH (1994) Responses of neurons in the 
parietal and temporal visual pathways during a motion task. J Neurosci 14:6171-6186. Medline

Girard P, Salin PA, Bullier J (1991) Visual activity in macaque area V4 depends on area 17 input. Neuroreport 2:81-84. CrossRef Medline

Goebel R, Muckli L, Zanella FE, Singer W, Stoerig P (2001) Sustained extrastriate cortical activation without visual awareness revealed by fMRI studies of hemianopic patients. Vision Res 41:1459-1474. CrossRef Medline

Harting JK, Huerta MF, Hashikawa T, van Lieshout DP (1991) Projection of the mammalian superior colliculus upon the dorsal lateral geniculate nucleus: organization of tectogeniculate pathways in nineteen species. J Comp Neurol 304:275-306. CrossRef Medline

Kato R, Takaura K, Ikeda T, Yoshida M, Isa T (2011) Contribution of the retino-tectal pathway to visually guided saccades after lesion of the primary visual cortex in monkeys. Eur J Neurosci 33:1952-1960. CrossRef Medline

Li P, Zhu S, Chen M, Han C, Xu H, Hu J, Fang Y, Lu HD (2013) A motion direction preference map in monkey V4. Neuron 78:376-388. CrossRef Medline

Lysakowski A, Standage GP, Benevento L (1988) An investigation of collateral projections of the dorsal lateral geniculate nucleus and other subcortical structures to cortical areas V1 and V4 in the macaque monkey: a double label retrograde tracer study. Exp Brain Res 69:651-661. Medline

Marrocco RT, Li RH (1977) Monkey superior colliculus: properties of single cells and their afferent inputs. J Neurophysiol 40:844-860. Medline

Maunsell JH, van Essen DC (1983) The connections of the middle temporal visual area (MT) and their relationship to a cortical hierarchy in the macaque monkey. J Neurosci 3:2563-2586. Medline

Mohler CW, Wurtz RH (1977) Role of striate cortex and superior colliculus in visual guidance of saccadic eye movements in monkeys. J Neurophysiol 40:74-94. Medline

Oostenveld R, Fries P, Maris E, Schoffelen JM (2011) FieldTrip: open source software for advanced analysis of MEG, EEG, and invasive electrophysiological data. Comput Intell Neurosci 2011:156869. CrossRef Medline

Rodman HR, Gross CG, Albright TD (1989) Afferent basis of visual response properties in area MT of the macaque: I. Effects of striate cortex removal. J Neurosci 9:2033-2050. Medline

Rodman HR, Gross CG, Albright TD (1990) Afferent basis of visual response properties in area MT of the macaque: II. Effects of superior colliculus removal. J Neurosci 10:1154-1164. Medline

Rodman HR, Sorenson KM, Shim AJ, Hexter DP (2001) Calbindin immunoreactivity in the geniculo-extrastriate system of the macaque: implications for heterogeneity in the koniocellular pathway and recovery from cortical damage. J Comp Neurol 431:168-181. CrossRef Medline

Roe AW, Chelazzi L, Connor CE, Conway BR, Fujita I, Gallant JL, Lu H, Vanduffel W (2012) Toward a unified theory of visual area V4. Neuron 74:12-29. CrossRef Medline

Schmid MC, Mrowka SW, Turchi J, Saunders RC, Wilke M, Peters AJ, Ye FQ, Leopold DA (2010) Blindsight depends on the lateral geniculate nucleus. Nature 466:373-377. CrossRef Medline

Sincich LC, Park KF, Wohlgemuth MJ, Horton JC (2004) Bypassing V1: a direct geniculate input to area MT. Nat Neurosci 7:1123-1128. CrossRef Medline

Tolias AS, Keliris GA, Smirnakis SM, Logothetis NK (2005) Neurons in macaque area $\mathrm{V} 4$ acquire directional tuning after adaptation to motion stimuli. Nat Neurosci 8:591-593. CrossRef Medline

Zeki S, Ffytche DH (1998) The Riddoch syndrome: insights into the neurobiology of conscious vision. Brain 121:25-45. CrossRef Medline 